\title{
Vorlagen für eine «Generaleinwilligung» und für ein Reglement
}

Michelle Salathé

Korrespondenz:

lic. iur. Michelle Salathé, MAE Schweizerische Akademie

der Medizinischen Wissenschaften SAMW Petersplatz 13

CH-4051 Basel Tel. 0612699030 Fax 0612699039

mail@samw.ch
2005 hat die Schweizerische Akademie der Medizinischen Wissenschaften (SAMW) die medizin-ethischen Richtlinien «Biobanken» veröffentlicht. Diese halten fest, dass die Gewinnung, Aufbewahrung und Nutzung von Proben für die Forschung nur mit Einwilligung des Spenders erfolgen darf. Um Spenderinnen und Spender nicht unnötig zu belasten und Forschungsvorhaben nicht durch komplizierte Einwilligungsprozeduren zu verzögern, wäre eine sogenannte Generaleinwilligung sinnvoll; allerdings müssten dafür bestimmte Voraussetzungen erfüllt sein.

Was ist unter einer «Generaleinwilligung» genau $\mathrm{zu}$ verstehen, und welche Voraussetzungen müssen erfüllt sein, damit diese den rechtlichen und ethischen Anforderungen an eine informierte Einwilligung entspricht? Eine Arbeitsgruppe*, initiiert von der Stiftung biobank-suisse, der SAMW und den Datenschutzbeauftragten der Kantone Zürich und Basel-Stadt, hat

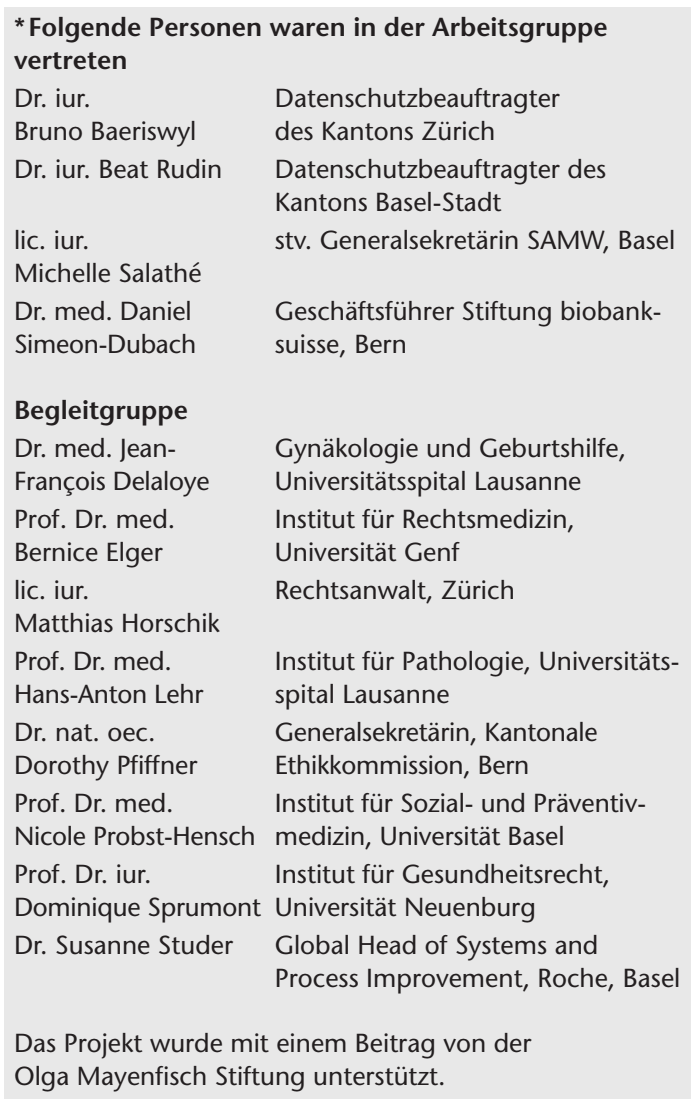

sich vertieft mit dem Thema auseinandergesetzt und Vorlagen zur Umsetzung der Generaleinwilligung ausgearbeitet.

\section{Umgang mit menschlichem biologischem Material in Schweizer Spitälern}

Um eine Ausgangsbasis für ihre Arbeiten zu haben, hat die Arbeitsgruppe im Mai 2008 eine Umfrage bei den Schweizer Spitälern zum Umgang mit menschlichem biologischem Material durchgeführt; die Direktionen von 220 Kliniken erhielten einen entsprechenden Fragebogen (gemäss Adressliste von $\mathrm{H}+$; ohne psychiatrische Kliniken und ohne Pflegeheime). Bis Ende August trafen 86 Antworten ein (Rücklaufquote $=39 \%$ ). Bei der Auswertung stellte sich heraus, dass einige der angeschriebenen Einrichtungen Tageskliniken oder andere spezialisierte Einrichtungen waren; die Antworten dieser Kliniken $(n=5)$ wurden von der Auswertung ausgeschlossen.

An der Umfrage beteiligt haben sich alle Universitätsspitäler sowie die Universitätskinderspitäler Basel, Bern und Zürich ( $\mathrm{n}=8), 15$ grosse Kantons- bzw. Regionalspitäler (d.h. mit mehr als 200 Betten), 19 mittelgrosse (mit 100 bis 200 Betten) und 24 kleine. Ausserdem haben 15 Privatkliniken den Fragebogen beantwortet.

Insgesamt 22 von 81 antwortenden Kliniken (27\%) gaben an, menschliches biologisches Material aufzubewahren, das für Forschung verwendet werden kann. Dies trifft - wenig überraschend - für alle Universitätsspitäler (inkl. Kinderspitäler) zu, sowie für 10 der 15 grossen Kantons- oder Regionalspitäler (66\%). Bei den mittleren (11\%) und kleinen Spitälern (4\%) sowie bei den Privatkliniken (7\%) waren die Werte wesentlich tiefer. Tabelle 1 zeigt, welche Arten von Material dies betrifft.

\section{Tabelle 1}

Art des aufbewahrten Materials $(n=22)$.

Blut 19 (86\%)

DNA/RNA

14 (64\%)

Gewebe

eingefroren

14 (64\%)

fixiert

12 (55\%)

Zellen

$13(59 \%)$

Anderes 3 (14\%) 
Angaben zur Grösse, Art und Qualität der Biobanken wurden nicht erhoben, um die Umfrage nicht zu überlasten. Aus dem gleichen Grund wurde auch nicht gefragt, wie viele Biobanken in den einzelnen Kliniken bestehen.

16 Spitäler $(73 \%)$, die angegeben hatten, menschliches biologisches Material für Forschungsprojekte aufzubewahren, geben dieses auch für spitalexterne Forschungsprojekte weiter (siehe Tabelle 2), meist an Forschende direkt (94\%), andere Spitäler (50\%), selten an Biobanken (5\%) oder die Industrie (4\%).

$95 \%$ der Spitäler geben an, die Patientinnen und Patienten über die Möglichkeit der Verwendung von humanem Material für Forschung $\mathrm{zu}$ informieren (z. B. allgemeine Spitalinformation, Spitalinformationsbroschüre, im Rahmen der Einwilligung für operative

\begin{tabular}{l}
$\begin{array}{l}\text { Tabelle } 2 \\
\text { Weitergabe des Materials }(n=16) \text {. }\end{array}$ \\
\begin{tabular}{ll} 
Universitätsspital $(n=8)$ & $6(75 \%)$ \\
\hline Grosses Kantons-, Regionalspital $(n=10)$ & $6(60 \%)$ \\
\hline Mittleres Kantons-, Regionalspital $(n=2)$ & $2(100 \%)$ \\
\hline Kleines Kantons-, Regionalspital $(n=1)$ & $1(100 \%)$ \\
\hline Privatspital $(n=1)$ & $1(100 \%)$
\end{tabular} \\
\hline
\end{tabular}

Eingriffe, Labor- oder Gewebeanalysen). Die Umfrage gibt keine Hinweise darauf, wie der Prozess der Einwilligung im Einzelnen ausgestaltet ist (Widerspruchslösung, Generaleinwilligung, spezifische Einwilligung usw.).

\section{Abbildung 1}

Muster für eine Einwilligungserklärung, die der Spender unterschreiben muss.

\section{Einwilligung}

Ich willige hiermit ein, dass

- meine Proben/Daten aufbewahrt werden, bis ich diese Einwilligung widerrufe;

- meine Proben/Daten, ohne dass ich darüber im konkreten Fall informiert werde und ohne dass dazu meine Einwilligung nochmals eingeholt werden muss, für zur Zeit unbestimmte biomedizinische Forschungsprojekte genutzt werden dürfen,

- wenn das Forschungsprojekt - sofern das anwendbare Recht die Bewilligungspflicht vorsieht - von der zuständigen Forschungsethikkommission bewilligt worden ist und

- wenn die Proben/Daten vor der Weitergabe so anonymisiert werden, dass die Beteiligten am Forschungsprojekt nicht wissen, von wem die Proben/Daten stammen,

- meine Proben/Daten an andere Biobanken weitergegeben werden dürfen,

- wenn gewährleistet ist, dass sie mindestens dieselben Standards für die Aufbewahrung in der Biobank einhalten, und

- wenn die Verantwortlichen der empfangenden Biobanken sich verpflichtet haben, bei der Weitergabe für Forschungsprojekte oder an andere Biobanken dieselben Voraussetzungen einzuhalten.

(Je nach Formulierung in der Informationsbroschüre, Wahlmöglichkeiten zum Umgang mit Ergebnissen, die zur Verhinderung, Feststellung und Behandlung bestehender oder künftig zu erwartender Krankheiten beitragen können (vgl. Varianten A und B in der Informationsbroschüre).

Ich bin einverstanden, dass die vorliegende Einwilligung über meinen Tod hinaus gilt.

Ich bestätige, dass

- ich hinreichend über die Aufnahme und Verwendung von Proben und Daten informiert wurde.

- ich die Möglichkeit hatte, Fragen zu stellen und allfällige Fragen zu meiner Zufriedenheit beantwortet wurden;

- ich darüber informiert wurde, dass meine Einwilligung freiwillig ist, dass mir also keine Vor- und Nachteile entstehen, wie ich mich auch entscheide.

(Informationen zur Biobank, zur verantwortlichen Organisation, zur [zur Zeit] verantwortlichen Person, mit einer Adresse für Fragen und Begehren [datenschutzrechtliche Rechtsansprüche, Widerrufsrecht], z.B. Website)

\section{(nötige Angaben zur Identifikation der Patientin/des Patienten)}

Ort, Datum, rechtsgültige Unterschrift (Bei Unmündigen oder Entmündigten muss die entsprechende Einwilligung der gesetzlichen Vertreterin/des gesetzlichen Vertreters vorliegen, bei urteilsfähigen Unmündigen zusätzlich deren persönliche Einwilligung.)

Ort, Datum, Unterschrift der Mitarbeiterin/des Mitarbeiter, welche(r) die Information vorgenommen und die Einwilligung eingeholt hat. 


\section{Vorlagen zur Umsetzung der Generaleinwilligung}

Die Zulässigkeit der Einwilligung in noch unbestimmte Forschungsprojekte per Generaleinwilligung ist juristisch nicht unumstritten; der Entwurf für das Bundesgesetz über die Forschung am Menschen sieht die Möglichkeit der Weiterverwendung von biologischem Material zu Forschungszwecken in den Artikeln 31 und 32 jedoch ausdrücklich vor. Aus Sicht der Arbeitsgruppe ist eine Generaleinwilligung unter nachfolgenden Voraussetzungen ein sinnvolles Instrument:

- die Spender haben die Einwilligung für die Weiterverwendung nach hinreichender Information freiwillig erteilt;

- die Spender wissen, dass sie ihre Einwilligung jederzeit widerrufen können;

- die Spender wissen, dass die Biobank, welche die Proben (und Daten) aufbewahrt, sich an Regeln hält, und diese in einem Reglement festgehalten hat (siehe Musterreglement Biobanken);

- die Spender wissen, dass der Datenschutz gewährleistet ist, namentlich, dass die Proben und Daten nur reversibel oder irreversibel kodiert weitergegeben werden;

- die Spender wissen, dass ein konkretes Forschungsvorhaben von der zuständigen Ethikkommission geprüft wird.

Für die Arbeitsgruppe stand die Suche nach praxistauglichen Lösungen im Vordergrund. Die Patienteninformation besteht demnach aus der «Einwilligungserklärung», die der Spender unterschreibt (siehe Abb. 1); sie wird durch die Patientenbroschüre ergänzt, welche die aus diversen Bereichen (Spitaljuristen, Leiter von Biobanken, Forschende usw.) diskutiert. Das Echo auf die Vorlagen war grundsätzlich positiv. Die Diskussionen haben aber auch gezeigt, dass die Probleme in der praktischen Umsetzung liegen: Wer soll die Generaleinwilligung einholen? Wann sollen die Patienten angesprochen werden? Genügt eine einmalige Generaleinwilligung oder muss bei jedem Spitaleintritt erneut nachgefragt werden? Wo wird die Einwilligung aufbewahrt und wie wird sichergestellt, dass der Widerruf der Einwilligung wirksam wird? Muss das Recht auf Wissen respektive auf Nichtwissen explizit erwähnt werden? Diese Fragen sind nicht einfach zu beantworten, und die Lösungsvorschläge fallen je nach Blickwinkel unterschiedlich aus. Weitere Aspekte, wie z.B. die Eigentumsrechte an den Proben oder Zugangsrechte, sind noch ungeklärt oder wenig geregelt.

Die Arbeitsgruppe empfiehlt den Spitälern, bereits heute die Patienten darüber aufzuklären, dass bei der Entnahme von Proben häufig therapeutisch und diagnostisch nicht benötigtes Material anfällt, das eine bedeutende Ressource für die medizinische Forschung darstellen kann. Falls die in diesem Artikel vorgestellten Vorlagen systematisch in den Schweizer Spitälern benutzt würden, könnten sich die Patienten klar dazu äussern, ob sie mit der Verwendung dieses überzähligen Materials zu Forschungszwecken einverstanden sind oder nicht. Dies wäre sowohl in Interesse der Patienten als auch der Forschenden in der Schweiz.

Ein starker Forschungsplatz Schweiz benötigt einfache und einheitliche Strukturen, die den administrativen Aufwand für die Forschung so klein als möglich halten. Um dem föderalistischen Wildwuchs ent-

\section{Die Diskussionen mit Expertinnen und Experten aus diversen Bereichen haben gezeigt, dass die Probleme in der praktischen Umsetzung liegen}

für den Spender relevanten Hintergrundinformationen enthält. Gleichzeitig hat die Arbeitsgruppe zwei Vorlagen für das Reglement von Biobanken ausgearbeitet; einerseits für Sammlungen von humanen Proben und Daten, die ausschliesslich für Forschungszwecke aufbewahrt werden, und andererseits für Sammlungen von Proben, die sowohl im Behandlungskontext aufbewahrt als auch für Forschung weiterverwendet werden (z. B. Probensammlungen in einem Institut für Pathologie).

Alle Vorlagen wurden Ende Mai 2009 anlässlich einer Veranstaltung mit Expertinnen und Experten gegenzuwirken, ist es daher wünschenswert, wenn die Spitäler diese Generaleinwilligung sowie - wo benötigt - das entsprechende Biobankenreglement zügig und ohne unnötige Änderungen implementieren. Es ist auch bereits geplant, die Anwendung dieser Dokumente in einem Begleitforschungsprojekt zu beobachten. Allenfalls auftretende Schwierigkeiten können so rasch identifiziert und korrigiert werden.

Die Vorlagen können von der Website der SAMW heruntergeladen werden (www.samw.ch). 